\title{
An Immunohistochemical Study on Expression of Laminin5 Gamma2 Chain and Alpha Smooth Muscle actin in Oral Squamous Cell Carcinoma Dr. Kavita Gupta ${ }^{1 *}$
}

${ }^{1}$ RGUHS, the Oxford Dental College, Bangalore, India

DOI: $\underline{10.36347 / \text { sjds.2022.v09i01.003 }}$

| Received: 14.12.2021 | Accepted: 17.01.2022 | Published: 28.01.2022

*Corresponding author: Dr. Kavita Gupta

RGUHS, the Oxford Dental College, Bangalore, India

Abstract

Original Research Article

Background and objectives: Head and neck carcinomas are the biological heterogeneous group of cancers, of which oral cancer is the most common. Ninety percent of oral cancers are squamous cell carcinomas originating from the mucosal epithelium carcinogenesis. Oral squamous cell carcinoma (OSCC) is one of the most prevalent cancers worldwide. The transformation of the normal epithelial cell into a tumor cell depends upon certain changes that happen at the cellular and molecular level which will help in its survival and proliferation. The basement membrane will show invasion by these altered tumor cells into the connective tissue stroma and then their subsequent spread and metastasis, which is an important prognostic indicator. Laminin-5 is a protein which is found to be associated with a migratory phenotype in epithelial neoplastic cells and also the stromal myofibroblasts play a key role in tumor invasion, due to its ability to modify the extracellular matrix. Aim: Laminin-5 (Ln-5) is the major component of the basement membrane and is involved for tumour progression. Alpha-smooth muscle actin ( $\alpha$-SMA) is an isoform of actin, positive in myofibroblasts and is an epithelial to mesenchymal transition (EMT) marker. EMT is a process by which tumor cells develop and are able to metastasize. Tumor cells progression is always followed by alteration in the cell composition and extracellular matrix . Therefore the aim of this study is to detect and evaluate the expression of laminin5 gamma 2 and $\alpha$-SMA in OSCC and to evaluate the association of density of stromal myofibroblasts with tumour budding intensity. Materials and methods: Thirty paraffin embedded tissue blocks of clinically diagnosed and histopathologically confirmed cases of OSCC were evaluated .In this we have divided them into 10 well differentiated OSCC, 10 moderately differentiated OSCC and 10 poorly differentiated OSCC for laminin-5 and $\alpha$ - smooth muscle actin (SMA) using standard immunohistochemistry technique. Semi-quantitative assessment of the expression of laminin and alpha SMA was done in all the study samples. The area of staining and the staining intensity was evaluated in order to determine the staining index which was then statistically analyzed between the three groups. The study data was analysed using SPSS (Statistical Package for Social Sciences) software V.22, IBM, Corp. Statistical Analysis was done using Chi Square test .The level of significance [P-Value] was set at $\mathrm{P}<0.01$. Results: There is a positive trend in high intensity expression of Lm5 $\gamma 2$ increasing with the histopathological grading of OSCC. This distribution of Lm5 $\gamma 2$ in varying grades of OSCC was significant $(\mathrm{p}=0.01 *)$. Expression of laminin was observed mainly around the tumor islands in OSCC group. $\alpha$ - SMA was seen with increasing intensity with increasing grade of the disease. Statistical comparison of $\alpha$ SMA in between the three groups using Kruskal- Wallis test showed a significant increase in the expression of $\alpha$-SMA in various grades of OSCC ( $\mathrm{p}<0.01)$. Conclusion: There is no strong association between expression of Lm5 $\gamma 2$ and $\alpha$-SMA. Independent association of each marker with histopathological grades of OSCC was seen in the study.

Keywords: Oral squamous cell carcinoma, Myofibroblasts, Smooth muscle actin, Immunohistochemistry.

Copyright $\odot 2022$ The Author(s): This is an open-access article distributed under the terms of the Creative Commons Attribution 4.0 International License (CC BY-NC 4.0) which permits unrestricted use, distribution, and reproduction in any medium for non-commercial use provided the original author and source are credited.

\section{INTRODUCTION}

Alpha-SMA is an actin isoform that plays a key role in process of fibrogenesis. Alpha-SMA can be found in smooth muscle cells, myofibroblasts, and in blood Vessels [1, 2] and can be correlated with activation of fibroblast to myofibroblast. The phenotype of myofibroblast in expression of $\alpha$-SMA and producing extracellular matrix is controlled by transforming growth factor-beta (TGF-beta) [3, 4].
Myofibroblast are associated with $\alpha$-SMA expression and are involved in inflammation, wound healing, fibrosis, and carcinogenesis [5]. Carcinoma cells that get transformed in to mesenchymal cells also express $\alpha$ SMA [6, 7]. Alpha-SMA, along with vimentin, Ecadherin, and fibronectin are the markers for the epithelial to mesenchymal transition (EMT) process. The EMT is a crucial step as it is involved in normal cells to become a cancerous cell [8]. In OSCC the 
extent of basement membrane (BM) defects correlates with invasive and metastatic potential [9-10]. The oral squamous cell carcinoma (OSCC) accounts for approximately $3 \%$ of all malignancies and more than $90 \%$ of cancers of the oral cavity and oropharynx [11]. OSCC is a neoplasm of epithelial origin with high prevalence rate in developing countries of the world especially in India [12]. Laminin-5 (Ln-5) is the major component of the basement membrane in most adult tissues and is a heterotrimer composed of three different laminin chains ( $\alpha 3, \beta 3$, and $\gamma 2$ chains). Formerly called as Kalinin, nicein, epiligrin or ladsin, laminin-5 is now designated as laminin 332 using a recently introduced simplified nomenclature [13-14]. The major functions of Ln-5 include the binding of epithelial cells to the basement membrane through the formation of hemidesmosomes and the migration of epithelial cells during the process of wound repair. In addition to this, Ln-5 has been implicated in tumor progression [15]. Several literature data have also suggested the potential role of laminin- 5 in OSCC [16, 17], breast cancer [18] and cervical adenocarcinoma [19] among others. Epithelial mesenchymal transition (EMT) is the process by which epithelial cells will adopt a mesenchymal phenotype or fibroblast-like properties and this transition of the neoplastic epithelial cells to acquire fibroblasts like properties is a significant event in the metastatic potential of the tumor [20]. The Myofibroblasts are the differentiated fibroblasts that express alpha smooth muscle actin ( $\alpha$ - SMA) and have intermediate characteristics among classic fibroblasts and smooth muscle cells. They have the ability to modify the extracellular matrix and hence actively participate in tumor invasion and metastasis [21]. Several studies have determined the role of myofibroblasts in OSCC [22-24]. Invasion of the altered tumor cells through the basement membrane into the connective tissue stroma and their subsequent spread and metastasis is an important prognostic indicator for understanding of this carcinogenesis model. Thus, the need of the present study is to evaluate the the expression of laminin 5 gamma 2 and $\alpha$-SMA in OSCC and to evaluate association of density of stromal myofibroblasts with tumour budding intensity for the better understanding of the carcinogenesis model for any therapeutic and prognostic implications.

\section{MATERIALS AND METHOD}

Thirty formalin fixed, paraffin embedded biopsy specimens with clinically and pathologically confirmed OSCC cases and samples were obtained from the Department of Oral and maxillofacial Pathology. Insufficient tissue lacking adequate depth, tissue specimen with inconclusive diagnosis, recurrent cases, cases with other concurrent oral lesions and previous history of therapy (surgery, chemotherapy and radiotherapy) were excluded from the study. The selected samples were then immunohistochemically analyzed for the expression of laminin-5 and $\alpha$ - SMA using standard techniques. Semi-quantitative assessment of the expression of laminin and alpha SMA was done in all the study samples. The area of staining and the staining intensity was evaluated in order to determine the staining index. The study data was analysed using SPSS (Statistical Package for Social Sciences) software V.22, IBM, Corp. Statistical Analysis was done using Chi Square test. The level of significance [P-Value] was set at $\mathrm{P}<0.01$.

\section{Haematoxylin and eosin staining}

Formalin fixed, paraffin embedded specimens were cut into $4 \mu \mathrm{m}$ sections and were stained with haematoxylin and eosin for histologic confirmation of clinical diagnosis of OSCC. Additional sequential sections were prepared for immunohistochemical studies.

\section{Immunohistochemistry protocol}

All the 30 cases of OSCC were available for high quality immunohistochemical staining. Immunohistochemical staining was performed on $4 \mu \mathrm{m}$ thick sections. All the procedures were performed at room temperature. The sections were deparaffinised through a series of xylene baths and rehydrated in graded concentrations of alcohol. Tissue sections were treated with $3 \%$ hydrogen peroxide to block endogenous peroxidase activity. Antigen retrieval was carried out by microwave with $0.01 \mathrm{M}$ sodium citrate buffer solution for tree cycles of $800 \mathrm{~W}$ for $5 \mathrm{~min}$ twice and $200 \mathrm{~W}$ for $14 \mathrm{~min}$ and later was subjected to two washes of tris buffer solution for $5 \mathrm{~min}$ each. Sections were then incubated with ready to use primary antibody (BIORBYT and BIOCARE). After washing with tris buffer solution, the sections were then incubated for 30 min with anti mouse secondary antibody and visualized using 3, 3'-diaminobenzidine (DAB) chromogen. Sections were then visualized under microscope (4X, $10 \mathrm{X}$ and 40X) magnification.

\section{The criteria used to define the $\operatorname{Lm} 5 \gamma 2$ positive for the IHC staining}

- Lm5 $\gamma 2$ staining found localized in epithelium, tumor cells and in the tumor nests in connective tissue stroma which appeared brownish in color.

- Round/ovoid brown stained cells which must be visible completely with cytoplasmic stain.

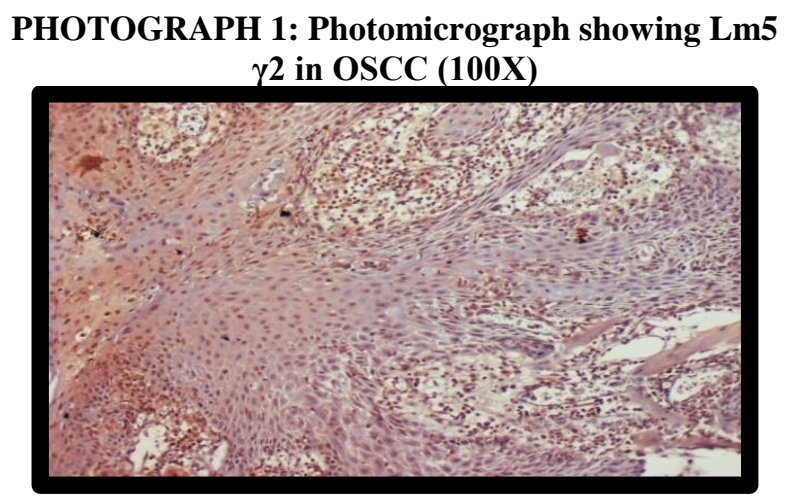


The criteria used to define the $\alpha$-SMA positive for the IHC staining

- $\quad \alpha-S M A$ staining found localized in large spindleshaped cells which are long slandered resembling bundles of microfilaments and appeared brownish in color in the connective tissue stroma.

PHOTOGRAPH 2: Photomicrograph showing $\alpha$ SMA in OSCC (100X)

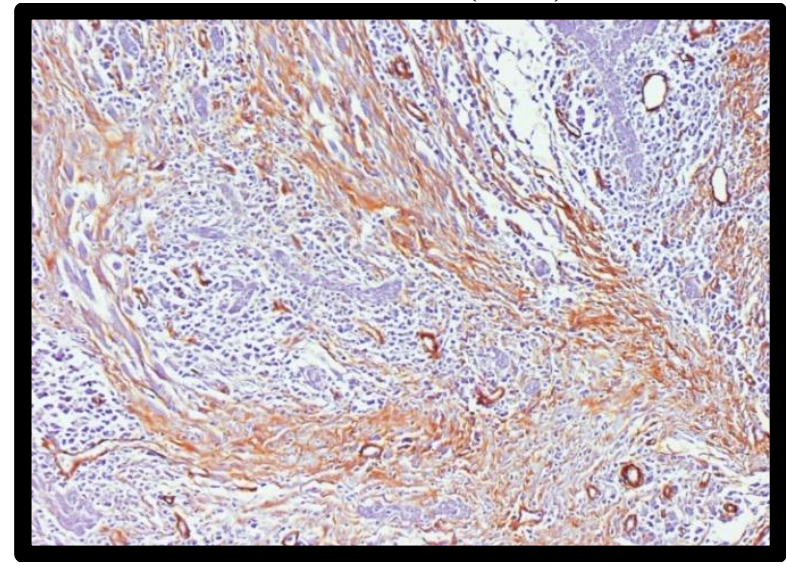

PHOTOGRAPH 3: BIORBYT Primary antibody used in the study $\operatorname{Lm5} \gamma 2$

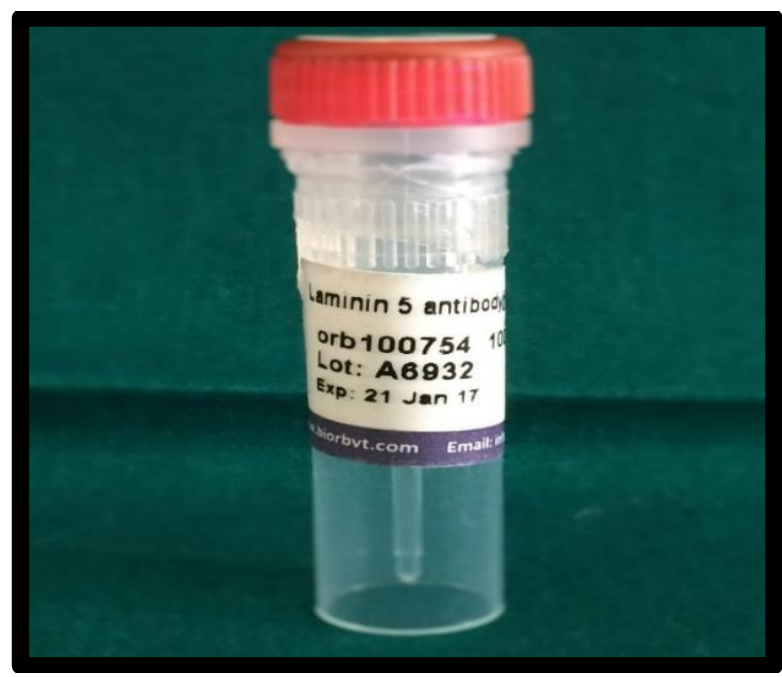

PHOTOGRAPH-4: BIOCARE primary antibody used in the study $\alpha-S M A$

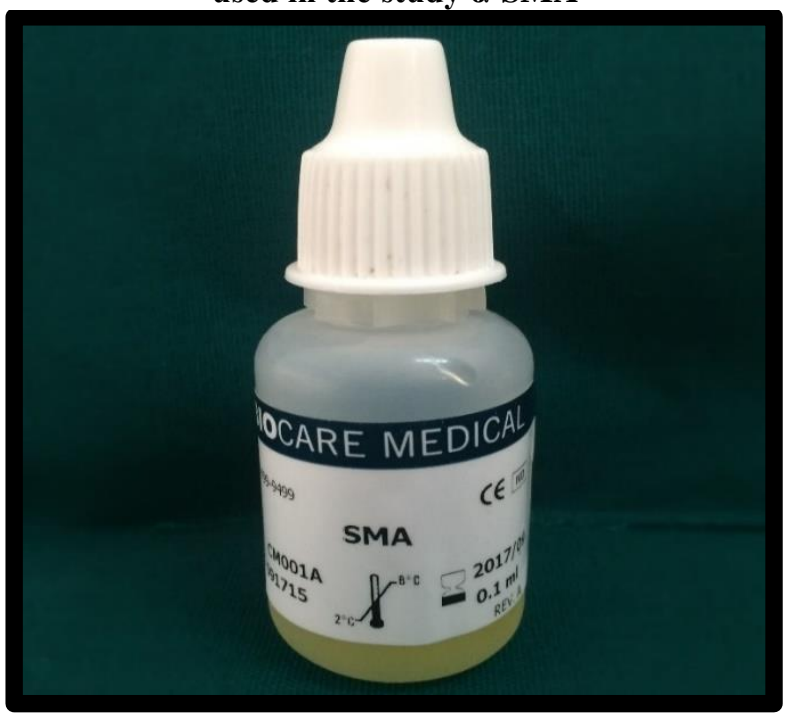

\section{RESULTS}

The present study was carried out to evaluate the expression of laminin 5 and stromal myofibroblasts in OSCC study groups. Semi-quantitative assessment of laminin and $\alpha$-SMA was done in all the cases. All the cases of laminin showed cytoplasmic staining mainly around the tumor islands in OSCC group. $\alpha$ - SMA was seen with increasing intensity along with the increasing grade of the disease. Staining index for both was calculated by the area of staining and intensity of staining as described in methodology earlier. Grade III, Lm5 $\gamma 2$ was majority in moderate intensity followed by high intensity with $30 \%$ and low intensity with $20 \%$ which shows there is a positive trend in high intensity expression of Lm5 $\gamma 2$ increasing with the histopathological grading of OSCC. This distribution of Lm5 $\gamma 2$ in varying grades of OSCC was significant $(\mathrm{p}=0.01 *)$. (table 1$)$.

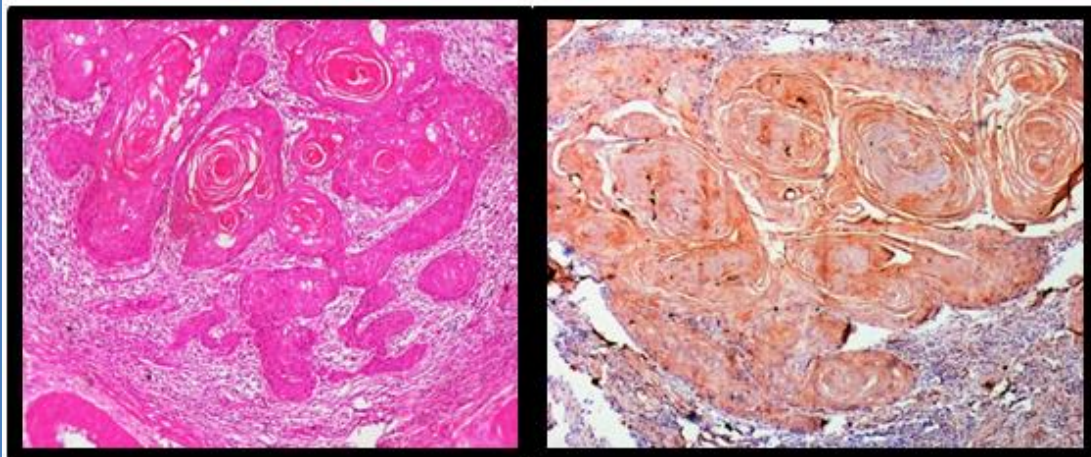

Haematoxylin and eosin staining
$\operatorname{Lm} 5 \gamma 2$ staining






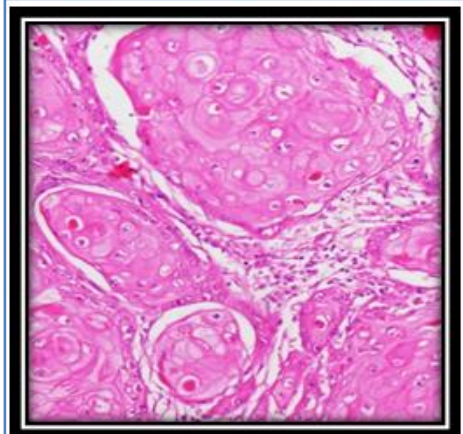

Haematoxylin and eosin staining

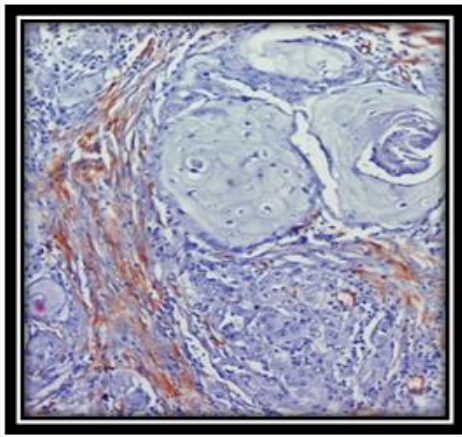

$\operatorname{Lm} 5 \gamma 2$ staining

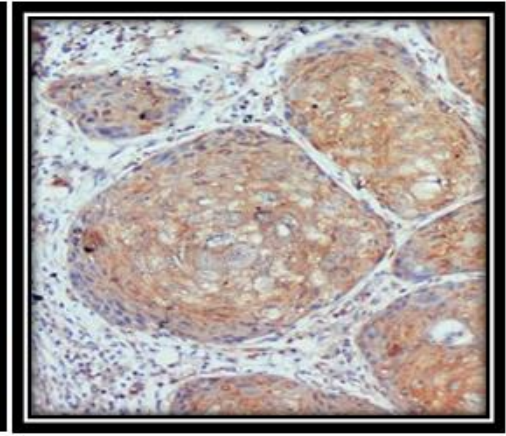

$\alpha-$ SMA staining

PHOTOMICROGRAPH (40 X ) OF POORLY DIFFERENTIATED OSCC

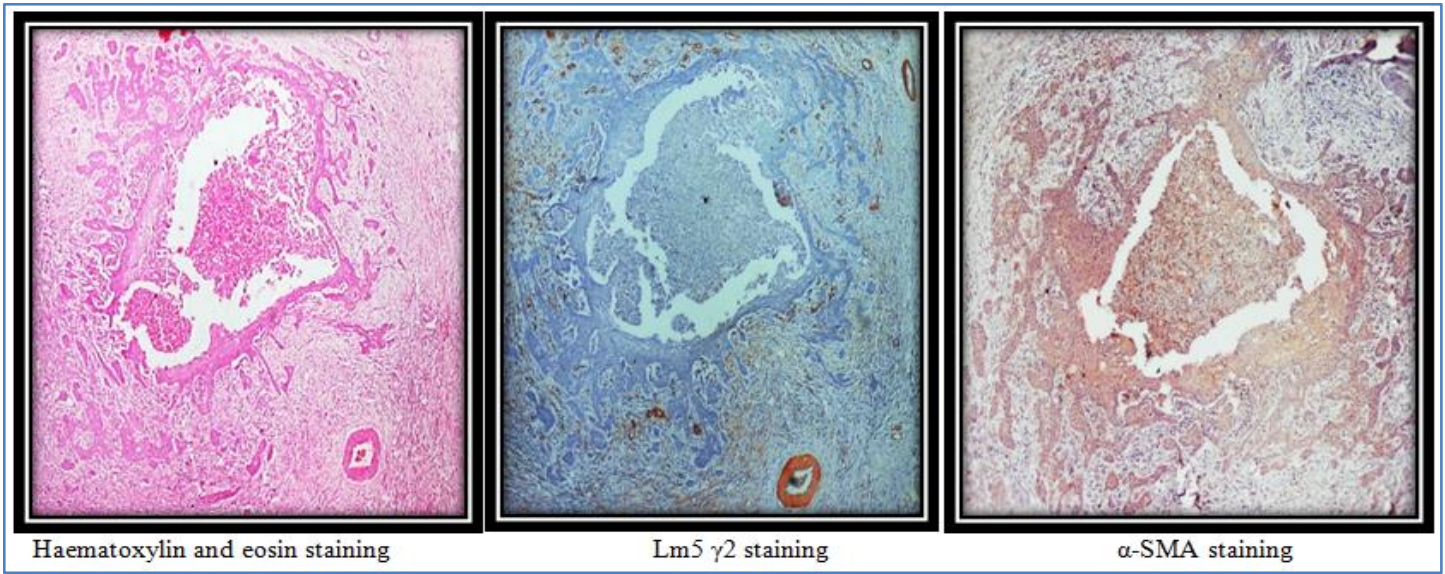

Evaluation of myofibroblasts through the immunohistochemical expression of $\alpha$-SMA revealed a low intensity in well differentiated OSCC, moderate intensity in moderately differentiated and intense staining in poorly differentiated OSCC especially around the tumor islands.

Table-1

\begin{tabular}{|c|c|c|c|c|c|c|c|c|}
\hline \multicolumn{9}{|c|}{ Comparison of the association of a - SMA and Lm5 $\gamma 2$ expressions using Chi Square test } \\
\hline \multirow[t]{2}{*}{$\operatorname{Lm5} \gamma 2$} & \multicolumn{2}{|c|}{ Negative } & \multicolumn{2}{|c|}{ Scanty } & \multicolumn{2}{|c|}{ Abundant } & \multirow[t]{2}{*}{$c^{2}$ Value } & \multirow[t]{2}{*}{ P-Value } \\
\hline & $\mathbf{n}$ & $\%$ & $\mathbf{n}$ & $\%$ & $\mathbf{n}$ & $\%$ & & \\
\hline Negative & 0 & $0.0 \%$ & 0 & $0.0 \%$ & 0 & $0.0 \%$ & \multirow[t]{4}{*}{3.591} & \multirow[t]{4}{*}{0.17} \\
\hline Low Intensity & 0 & $0.0 \%$ & 7 & $46.7 \%$ & 4 & $26.7 \%$ & & \\
\hline Mod. Intensity & 0 & $0.0 \%$ & 5 & $33.3 \%$ & 3 & $20.0 \%$ & & \\
\hline High Intensity & 0 & $0.0 \%$ & 3 & $20.0 \%$ & 8 & $53.3 \%$ & & \\
\hline
\end{tabular}

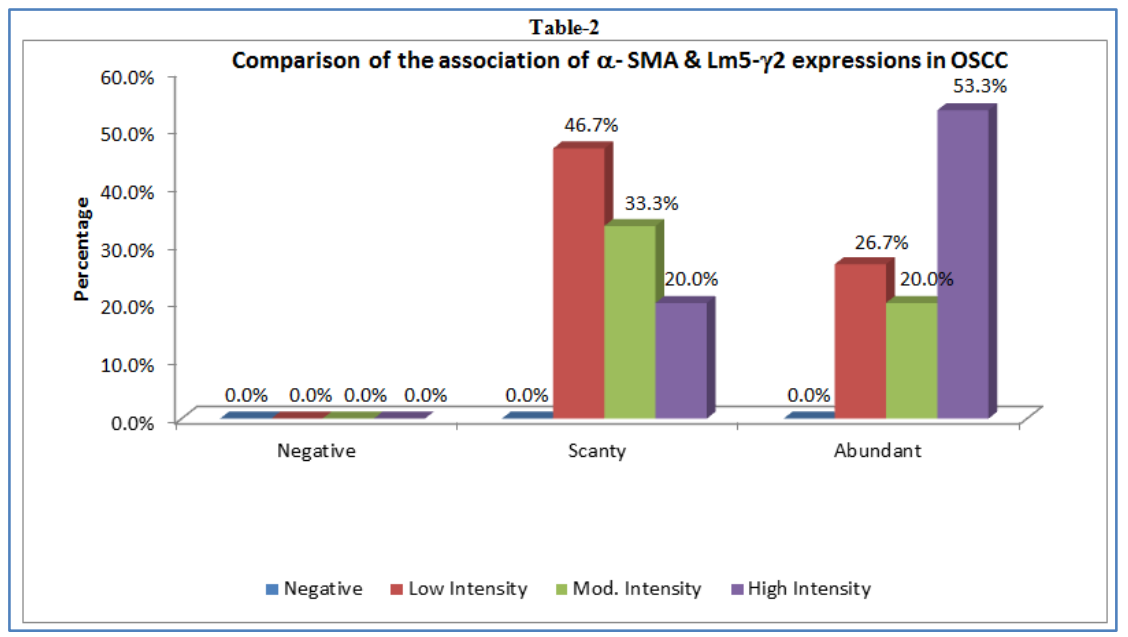


Additionally, regression analysis was done to evaluate the strength of association between $\alpha$-SMA and laminin in the three groups. The analysis (table-2) revealed statistical significance in OSCC and the association was not significant $(\mathrm{p}=0.17)$. This infers as the $\alpha$-SMA expression increases, relative to the histopathological grading of OSCC, the Lm5 $\gamma 2$ also increases correspondingly. However, the association with the expressions of $\alpha$-SMA(density of stromal myofibroblasts) and Lm5 $\gamma 2$ for varying histopathological gradings of OSCC was not statistically significant $(\mathrm{p}=0.17)$.

\section{DISCUSSION}

The study results were in accordance with several other results obtained from the literature [2526]. Progressive decrease in laminin expression in OSCC could be attributed to the E-cadherin expression loss. The altered distribution of laminin and collagen IV are associated with the progression of OSCC [27].

In present study the $\alpha$-SMA expression for myofibroblasts were found to be increasing with increased grade of OSCC and was statistically significant. This was in accordance with various studies [28, 29]. In OSCC, an increase in myofibroblasts population causes proteolytic degradation of the stromal components thereby aiding in the spread of tumor cells. Additionally, myofibroblasts may contribute to cytokine production and tumor angiogenesis. The stromal reaction to the altered overlying epithelium varies from increased inflammatory response, altered collagen fiber remodelling and vasculature and to some extent to the presence of myofibroblasts. The possible reason for an increased myofibroblasts is that that the genetically altered epithelium may have an inductive effect on the adjacent stroma to produce myofibroblasts [22]. Additionally, the changes in the composition and organization of the stromal micro-environment associated with the cytokine release may aid in the formation of myofibroblasts [30]. The exact role of these cells however is unknown.

Etemad-Moghadam et al. (2009) showed that the presence of myofibroblasts was significantly higher in oral squamous cell carcinomas compared to both dysplasia and normal mucosa. These findings show the presence of myofibroblasts in the stroma of oral squamous cell carcinoma but not in dysplasia and normal mucosa, suggesting further investigation to clarify the role of myofibroblasts in the carcinogenesis process [22]. Chaudhary et al. (2012) found an increase expression as the disease progresses from oral premalignancy to verrucous carcinoma and to invasive OSCC thereby suggesting that the proliferation of myofibroblasts may be used as a stromal marker for premalignancy and malignancy [29]. Analysis of $\alpha$ SMA expression for myofibroblasts proliferation can be used as a stromal marker for predicting behaviour in oral pre-cancer and cancer.
The Trans differentiation of oral fibroblasts to myofibroblasts could occur secondary to the release of transforming growth factor- $\beta 1$ that is released by OSCC cells. Analysis of myofibroblasts expression in varying grades of OSCC may indicate that the loss of cellular differentiation affects the number of myofibroblasts in the tumor stroma [23, 28]. Stromal destruction in OSCC occurs either through cancer cell-prompted destruction or due to cooperation between cancer cell and surrounding stroma. Myofibroblast appearance in invasive cancer and tumor desmoplasia are important reflection of the tumor-host interaction, especially in aggressive cancers. The Trans differentiation of myofibroblasts is generally induced during the invasive stage of SCC and further loss of tumor differentiation would not affect the number of these cells [30].

Statistical correlation of laminin-5 and alphaSMA in oral epithelial dysplasia and OSCC showed an inverse relationship between the two wherein the decreased expression of laminin was associated with an increase in myofibroblasts population. Franz et al. (2010) demonstrated that increased laminin chain deposition is associated with a rising ASMA positivity suggesting a special role of stromal myofibroblast transdifferentiation for laminin matrix remodelling in OSCC. Laminin $\alpha 2$ chain significantly decreased while $\alpha 3, \alpha 4, \alpha 5$ and $\gamma 2$ chains and $\alpha$-smooth muscle actin (ASMA) significantly increase with rising grade of oral squamous cell carcinoma [31]. Similar kind of result was noted in our study. It was hypothesized that mediated by myofibroblasts, OSCC development is associated with a stromal upregulation of laminin isoforms possibly contributing to a migration promoting microenvironment. It is possible that mesenchymal cells contribute to the promotion of tumor cell migration as well as vessel formation in OSCC by providing and organizing pro-migratory laminin-5 fragment [32]. Epithelial-mesenchymal interaction plays a key role in oral carcinogenesis. An inverse relationship between laminin 5 and $\alpha$-SMA expression probably suggest that the epithelial and mesenchymal components have a synergistic effect for tumor invasion. Terashi et al. in there study told that the Laminin-5 c2 expression was detected in the cytoplasm of tumor cells, predominantly in single cells or in the cells of tumor nests and epithelium. Expression of laminin-5 c2 was reported in several epithelial malignant tumors. Our study showed that Grade III, Lm5 $\gamma 2$ was majority in moderate intensity followed by high intensity with $30 \%$ and low intensity with $20 \%$ which shows there is a positive trend in high intensity expression of $\operatorname{Lm} 5 \gamma 2$ increasing with the histopathological grading of OSCC. This distribution of Lm5 $\gamma 2$ in varying grades of OSCC was significant $(\mathrm{p}=0.01 *)$.

\section{CONCLUSION}

There is no strong association between expression of Lm5 $\gamma 2$ and $\alpha$-SMA and show an independent association of each marker with 
histopathological grades of OSCC. Future study should also focus on $\alpha$-SMA as a prognostic marker for targeted therapy as it is one of the epithelial to mesenchymal marker. Laminin can be adopted as a useful marker in evaluating the histological differentiation and aggressiveness of oral carcinoma. The increased expression of $\alpha$-SMA suggests that the proliferation of myofibroblasts may be used as a prognostic marker for malignancy. Additionally, myofibroblasts could be useful as a potential target for chemotherapeutic regimen in oral squamous cell carcinoma.

\section{REFERENCES}

1. R Cherng, S., Young, J., Ma, H. (2008). Alphasmooth muscle actin ( $\alpha$-SMA). J Am Sci, 4, 10. 2. Feller L, Lemmer J. Oral squamous cell carcinoma: epidemiology, clinical presentation and treatment. Journal of cancer therapy 2012; 3:263

2. Shinde, A.V., Humeres, C., Frangogiannis, N.G. (2016). The role of alpha-smooth muscle actin in fibroblast-mediated matrix contraction and remodeling. Biochim Biophys Acta, 1863, 298309.

3. Grotendorst, G.R., Rahmanie, H., Duncan, M.R. (2004). Combinatorial signaling pathways determine fibroblast proliferation and myofibroblast differentiation. FASEB J, 18, 46979.

4. Sun, K.H., Chang, Y., Reed, N.I. (2016). AlphaSmooth muscle actin is an inconsistent marker of fibroblasts responsible for force-dependent TGFbeta activation or collagen production across multiple models of organ fibrosis. Am J Physiol Lung Cell Mol Physiol, 310, 824-36.

5. Rao, K.B., Malathi, N., Narashiman, S. (2014). Evaluation of myofibroblasts by expression of alpha smooth muscle actin: a marker in fibrosis, dysplasia and carcinoma. J Clin Diagn Res, 8, ZC14-7.

6. Kalluri, R., \& Weinberg, R. A. (2009). The basics of epithelial-mesenchymal transition. The Journal of clinical investigation, 119(6), 1420-1428.

7. Enescu, A., Enescu, A. Ş., Florou, C., \& Petrescu, F. (2014). E-cadherin and $\alpha$-SMA expression in the epithelial-mesenchymal transition of salivary glands pleomorphic adenomas. Romanian journal of morphology and embryology= Revue roumaine de morphologie et embryologie, 55(4), 1383-1387.

8. Kalluri, R., \& Weinberg, R. A. (2009). The basics of epithelial-mesenchymal transition. The Journal of clinical investigation, 119(6), 1420-1428.

9. Han, Y. H., Gao, B., Huang, J. H., Wang, Z., Guo, Z., Jie, Q., \& Luo, Z. J. (2015). Expression of CD147, PCNA, VEGF, MMPs and their clinical significance in the giant cell tumor of bones. International journal of clinical and experimental pathology, 8(7), 8446.

10. Kobayashi, H., Tsuruchi, N., Sugihara, K., Kaku, T., Saito, T., Kamura, T., \& Taniguchi, S. I. (1993).
Expression of $\alpha$-smooth muscle actin in benign or malignant ovarian tumors. Gynecologic oncology, 48(3), 308-313.

11. Rao, S. V. K., Mejia, G., Roberts-Thomson, K., \& Logan, R. (2013). Epidemiology of oral cancer in Asia in the past decade-an update (20002012). Asian Pacific journal of cancer prevention, 14(10), 5567-5577.

12. Feller, L., \& Lemmer, J. (2012). Oral squamous cell carcinoma: epidemiology, clinical presentation and treatment.

13. Lohi, J. (2001). Laminin-5 in the progression of carcinomas. International journal of cancer, 94(6), 763-767.

14. Aumailley, M. (2013). The laminin family. Cell Adh Migr, 7; 48-55

15. Miyazaki, K. (2006). Laminin-5 (laminin-332): unique biological activity and role in tumor growth and invasion. Cancer science, 97(2), 91-98.

16. Lenander, C., Habermann, J. K., Öst, Å., Nilsson, B., Schimmelpenning, H., Tryggvason, K., \& Auer, G. (2001). Laminin-5 $\gamma 2$ chain expression correlates with unfavorable prognosis in colon carcinomas. Analytical Cellular Pathology, 22(4), 201-209.

17. Yellapurkar, S., Natarajan, S., Boaz, K., Manaktala, N., Baliga, M., Shetty, P., \& Ravi, M. (2018). Expression of laminin in oral squamous cell carcinomas. Asian Pacific journal of cancer prevention: APJCP, 19(2), 407.

18. Qiu, X., Tan, H., Fu, D., Zhu, Y., \& Zhang, J. (2018). Laminin is over expressed in breast cancer and facilitate cancer cell metastasis. Journal of cancer research and therapeutics, 14(12), 1170.

19. Imura, J., Uchida, Y., Nomoto, K., Ichikawa, K., Tomita, S., Iijima, T., \& Fujimori, T. (2012). Laminin-5 is a biomarker of invasiveness in cervical adenocarcinoma. Diagnostic pathology, 7(1), 1-5.

20. Brabletz, T., Hlubek, F., Spaderna, S., Schmalhofer, O., Hiendlmeyer, E., Jung, A., \& Kirchner, T. (2005). Invasion and metastasis in colorectal cancer: epithelial-mesenchymal transition, mesenchymal-epithelial transition, stem cells and $\beta$-catenin. Cells tissues organs, 179(1-2), 56-65.

21. de-Assis, E. M., Pimenta, L. G., Costa-e-Silva, E., Souza, P. E., \& Horta, M. C. (2012). Stromal myofibroblasts in oral leukoplakia and oral squamous cell carcinoma. Medicina oral, patologia oral y cirugia bucal, 17(5), e733.

22. Etemad-Moghadam, S., Khalili, M., Tirgary, F., \& Alaeddini, M. (2009). Evaluation of myofibroblasts in oral epithelial dysplasia and squamous cell carcinoma. Journal of oral pathology \& medicine, 38(8), 639-643.

23. Kawashiri, S., Tanaka, A., Noguchi, N., Hase, T., Nakaya, H., Ohara, T., \& Yamamoto, E. (2009). Significance of stromal desmoplasia and myofibroblast appearance at the invasive front in 
squamous cell carcinoma of the oral cavity. Head \& Neck: Journal for the Sciences and Specialties of the Head and Neck, 31(10), 1346-1353.

24. Joshi, P. S., Patil, J., Chougule, M., Dudanakar, M., \& Hongal, B. P. (2016). Evaluation of stromal myofibroblasts in epithelial dysplasia and oral squamous cell carcinoma: An immunohistochemical study. Clinical Cancer Investigation Journal, 5(5), 441.

25. Yellapurkar, S., Natarajan, S., Boaz, K., Manaktala, N., Baliga, M., Shetty, P., \& Ravi, M. (2018). Expression of laminin in oral squamous cell carcinomas. Asian Pacific journal of cancer prevention: APJCP, 19(2), 407.

26. Shruthy, R., Sharada, P., Swaminathan, U., \& Nagamalini, B. R. (2013). Immunohistochemical expression of basement membrane laminin in histological grades of oral squamous cell carcinoma: A semiquantitative analysis. Journal of oral and maxillofacial pathology: JOMFP, 17(2), 185.

27. Diniz-Freitas, M., García-Caballero, T., AntúnezLópez, J., Gándara-Rey, J. M., \& García-García, A. (2006). Reduced E-cadherin expression is an indicator of unfavourable prognosis in oral squamous cell carcinoma. Oral oncology, 42(2), 190-200.

28. Bello, I. O., Vered, M., Dayan, D., Dobriyan, A., Yahalom, R., Alanen, K., \& Salo, T. (2011).
Cancer-associated fibroblasts, a parameter of the tumor microenvironment, overcomes carcinomaassociated parameters in the prognosis of patients with mobile tongue cancer. Oral oncology, 47(1), 33-38.

29. Chaudhary, M., Gadbail, A. R., Vidhale, G., Mankar, M. P., Gondivkar, S. M., Gawande, M., \& Patil, S. (2012). Comparison of myofibroblasts expression in oral squamous cell carcinoma, verrucous carcinoma, high risk epithelial dysplasia, low risk epithelial dysplasia and normal oral mucosa. Head and neck pathology, 6(3), 305-313.

30. Gaggioli, C. (2008). Collective invasion of carcinoma cells: when the fibroblasts take the lead. Cell adhesion \& migration, 2(1), 45-47.

31. Franz, M., Wolheim, A., Richter, P., Umbreit, C., Dahse, R., Driemel, O., \& Berndt, A. (2010). Stromal laminin chain distribution in normal, hyperplastic and malignant oral mucosa: relation to myofibroblast occurrence and vessel formation. Journal of oral pathology \& medicine, 39(4), 290-298.

32. Franz, M., Hansen, T, Borsi, L., Geier, C., Hyckel, P., Schleier, P. (2007). A quantitative colocalization analysis of large unspliced tenascin-CL and laminin-5/ 2 -chain in basement membranes of oral squamous cell carcinoma by confocal laser scanning microscopy. J Oral Pathol Med, 36; 6-11. 